\title{
Sistematização da assistência de Enfermagem na perspectiva da equipe de enfermagem de um hospital público do norte do Brasil
}

RESUMO | Objetivo: Trata-se de estudo o qual tem como foco compreender o entendimento da equipe de enfermagem sobre a sistematização da assistência de enfermagem. Método: Pesquisa descritiva, de abordagem qualitativa realizado com 40 profissionais de enfermagem de uma maternidade referência da Região Central de Belém, com a utilização de entrevista semi-estruturada e realizada a análise de conteúdo de Bardin. A pesquisa foi aprovada pelo comitê de ética sob o parecer $n^{\circ} 547.872$. Resultados: Houve três categorias temáticas, com base na análise de conteúdo, a partir do qual se observou que os participantes possuem conhecimento insuficiente para aperfeiçoar a implementação do processo de enfermagem e consequentemente tais podem refletir na assistência. Conclusão: O entendimento da equipe de enfermagem é insuficiente para concretizar a SAE na assistência adjunto das dificuldades presentes no ambiente hospitalar.

Palavras-chaves: Processo de enfermagem; Equipe de Enfermagem; Maternidades.

ABSTRACT | Objective: This study focuses on understanding the nursing team's understanding of the systematization of nursing care. Method: Descriptive research, with a qualitative approach conducted with 40 nursing professionals from a reference maternity in the Central region of Belém, with the use of semi-structured interviews and performed the content analysis of Bardin. The research was approved by the Ethics committee under the $N^{\circ}$ 547.872. Results: There were 3 thematic categories, based on content analysis, from which it was observed that the participants have insufficient knowledge to optimize the implementation of the nursing process and consequently, they may reflect on the assistance. Conclusion: The understanding of the nursing team is insufficient to achieve the SAE in the adjunctive assistance of the difficulties present in the hospital environment.

Keywords: Nursing process; Nursing; Team; Hospitals; Maternity.

RESUMEN | Objetivo: Este estudio se centra en entender la comprensión del equipo de enfermería de la sistematización de la atención de enfermería. Método: Investigación descriptiva, con un enfoque cualitativo realizado con 40 profesionales de enfermería de una maternidad de referencia en la región central de Belém, con el uso de entrevistas semiestructuradas y realizado el análisis de contenido de Bardin. Resultados: Había 3 categorías temáticas, basadas en el análisis del contenido, de las que se observó que los participantes no tienen conocimientos suficientes para optimizar la implementación del proceso de enfermería y, en consecuencia, estos pueden reflexionar sobre la asistencia. Conclusión: La comprensión del equipo de enfermería es insuficiente para lograr el NCS en la asistencia adjunta de las dificultades presentes en el entorno hospitalario.

Palavras claves: Proceso de Enfermería; Grupo de Enfermería; Maternidades.

\section{Fabio Conceição dos Santos}

Enfermeiro especialista em atenção a neurologia e terapia intensiva e neonatal.

\section{Jaqueline Dantas Neres Martins}

Graduanda da Universidade do Estado do Pará (UEPA).

\section{Marcelo Williams Oliveira de Souza}

Doutorando em doenças infecciosas pela Universidade Federal do Pará (UFPA).

\section{Dayara de Nazaré Rosa de Carvalho}

Enfermeira e docente da Faculdade

Paraense de Ensino (FAPEN).

\section{Annália de Paula Gesta Santos}

Enfermeira.

\section{Ingrid Magali de Souza Pimentel}

Enfermeira. Doutora em processos educacionais. 
INTRODUÇÃO

A assistência da enfermagem requer métodos e instrumentos os quais fundamentem e direcionem as melhores estratégias de ações voltadas para a promoção a saúde, prevenção de doenças e agravos e reabilitação da saúde ${ }^{1}$. Nesse ínterim, há um conjunto de regulamentos jurídicos os quais embasam e oferecem notoriedade a necessidade de planejamento e programação dos serviços de enfermagem a fim de melhor sistematizar e promover a resolutividade dos problemas de saúde do indivíduo, família e comunidade ${ }^{2-3}$.

Entre um dos aspectos que norteiam esse planejamento está a obrigatoriedade da Sistematização da Assistência de Enfermagem (SAE) e do Processo de Enfermagem (PE) nas instituições públicas e privadas em toda e qualquer assistência prestada pelo enfermeiro. Sendo de extrema valia o conhecimento sobre a diferença entre SAE e PE, a inter-relação entre ambas bem como a significância dos mesmos para a categoria profissional, segurança e qualidade dos serviços prestados ${ }^{3-4}$.

A SAE é uma metodologia científica que guia e orienta as ações de enfermagem a fim de proporcionar cientificidade, melhor tomada de decisão e impulsionar a qualidade administrativa- gerencial no cuidado de enfermagem, a qual configura a materialização da assistência de enfermagem em seus aspectos mais amplos e organizacionais. O PE corresponde ao fornecimento de assistência ao paciente mediante plano de cuidado construído e anexados em etapas intercaladas embasado em julgamento clínico adjunto do suporte teórico ${ }^{1}$.

Inúmeros estudos denotam as contribuições que a SAE propicia ao sistema de saúde, ao enfermeiro e paciente, a saber: organização do cuidado, norteamento das decisões, possibilidade de averiguar os resultados, dinamização da assistência, qualidade e segurança do cuidado, continuidade da assistência, autonomia do profissional de enfermagem, menor tempo de internação do paciente e resolutividade nos problemas de saúde $\mathrm{e}^{3-6}$.

No entanto, apesar dos usufrutos trazidos pela $\mathrm{SAE}$, bem como da obrigatoriedade legal, existem dificuldades na sua implementação nas Instituições de Saúde, devido a multifatores, dentre eles o mais recorrentes é a falta de conhecimento da equipe de enfermagem sobre a aplicabilidade desta e seus benefícios. Este fator comumente pode favorecer uma assistência deficitária e despida de cientificidade, em que os problemas de saúde podem ser cíclicos e passíveis de maiores complicações e transtornos ${ }^{4-5}$.

Em virtude dessas deficiências na implementação da SAE, demonstrada por inúmeras pesquisas, e das consequências disto, é importante estudos como este, que visem aprofundar os fatores causais que dificultam a estabilização da SAE e $\mathrm{PE}$, a fim de elaborar estratégias satisfatórias e voltadas a resolução dos mesmos.

Considerando também que, o setor de maternidade é um ambiente, na qual frequentemente há déficits na implementação da SAE, além de que, contem complexidade tecnológica-assistencial por abarcar gestantes, nutrizes e puérperas de variados riscos, que necessitarão de monitorização, assistência contínua por determinado tempo, educação em saúde, autocuidado. Para que tais sejam realizados é necessário o dimensionamento de recursos, ferramentas e estratégias por parte da equipe de enfermagem através da SAE.

Na região norte, este estudo traz contribuições, já que é um dos pioneiros a realizar tal pesquisa, na qual anexo a isso, coexistem particularidades regionais e culturais específicas principalmente, no que tange ao processo de nascimento e amamentação que podem contribuir ou ser empecilhos no contexto da assistência. $E$, portanto, estes devem ser abarcados no contexto de planejamento da SAE, a fim de assegurar a integralidade do cuidado.

Dessa maneira, é importante compreender se a equipe de enfermagem possui conhecimento sobre $\mathrm{SAE}$ e $\mathrm{PE}$, já que o conhecimento teórico-prático e a credi- bilidade da equipe de enfermagem em tal é um dos pilares fundamentais para a implementação ${ }^{6,7}$. Neste sentido, a pergunta do estudo é qual a compreensão da equipe de enfermagem sobre Sistematização da Assistência de Enfermagem? Como objetivo elencou-se: Compreender o entendimento da equipe de enfermagem sobre a sistematização da assistência de enfermagem em um hospital público de referência no Norte do País.

\section{MÉTODO}

Trata-se de um estudo exploratório, descritivo com abordagem qualitativa realizado na maternidade referência da Região Central de Belém. Participaram desta pesquisa 40 enfermeiros (as) e técnicos (as) de enfermagem, tendo como critérios de inclusão: ser enfermeiro e/ou técnico de enfermagem e trabalhar mínimo de um ano nas enfermarias do local.

Utilizou-se uma entrevista semi-estruturada, cujo objetivo foi conhecer o entendimento da equipe de enfermagem sobre a temática. $\mathrm{O}$ instrumento de pesquisa continha as seguintes perguntas sobre: Você sabe o que é SAE? Como você define SAE? Você aplica SAE nesta unidade? Quais as etapas da SAE que você aplica? Quais os benefícios da SAE para o paciente? Quais os benefícios da SAE para o cuidado de enfermagem? A SAE é aplicada no seu plantão?, caso não explicar por que.

O material obtido neste processo foi interpretado a base da análise de conteúdo de Bardin, da qual emergiram as seguintes categorias: 1. Entendimento da equipe de enfermagem sobre a Sistematização da Assistência de Enfermagem. 2. Entendimento da equipe de enfermagem quanto aos benefícios da SAE para os pacientes. 3 . Entendimento dos benefícios da SAE como um método que organiza a assistência e dá qualidade nos cuidados de enfermagem.

Este estudo foi aprovado pelo Comitê de ética em pesquisa da Escola de Enfermagem Magalhães Barata - UEPA/CAMPUS IV sob o número do parecer 547.872 e CAAE 27559214.0.00005170. 
No momento da pesquisa, os participantes foram informados, sobre o termo de consentimento livre e esclarecido (TCLE), assim como os riscos e benefícios advindos da pesquisa, bem como as medidas cabíveis para minimização desses riscos. Como por exemplo, o sigilo de todas as informações, em respaldo aos aspectos éticos em pesquisas que envolvem os seres humanos. Para manter o anonimato dos participantes utilizou-se o sistema alfanumérico (E1, E2, E3,...); cuja letra inicial significada "Enfermeira" e (TE1, TE2, TE3,...); para "Técnica de Enfermagem" usando apenas as iniciais seguidas de um número ordinal para identificar os participantes.

\section{RESULTADOS}

A pesquisa foi realizada com 40 participantes dos quais, 20 eram enfermeiros e 20 técnicos de enfermagem.

Em relação aos participantes do estudo $100 \%$ eram do sexo feminino, mesmo que a priori, este não fosse um critério de inclusão. Em relação às enfermeiras, a idade variou de 36 a 57 anos, com tempo de formação de 10 a 30 anos. Destas, 19 possuem pós-graduação específica em obstetrícia e/ou em outras áreas.

O tempo de trabalho dos enfermeiros no local variou de 2 anos e 3 meses até 30 anos.

Quanto aos técnicos de enfermagem a idade variou entre 29 a 63 anos, com tempo de formação técnica de 6 a 28 anos. Apenas 2 técnicas de enfermagem possuem curso voltado para a obstetrícia.

Entendimento da equipe de enfermagem sobre a Sistematização da Assistência de Enfermagem

$\mathrm{Na}$ pesquisa é evidenciado que os enfermeiros conhecem pouco sobre SAE. Teoricamente compreendem que a SAE é uma atribuição privativa do enfermeiro, construída e dimensionada sobre problemas reais do paciente adjunto das vulnerabilidades com o objetivo de otimizar as respostas da família, indivíduo ou comunidade $^{4}$. Os participantes reconhecem-na como ferramenta metodológica assistencial de organização e dimensionamento da assistência de enfermagem, pautado nas necessidades individuais do paciente. Além disso, é evidenciado também em uma das falas que a aplicabilidade da SAE depende de conhecimento técnico-científico para a sua efetivação ${ }^{5}$.

É parte do processo de enfermagem que visa uma assistência de qualidade e baseada em conhecimentos técnicos e científicos, assim seguindo uma sequência ou etapas desde a admissão até a alta do paciente. (E11).

[...] organização da execução do processo de enfermagem, com uma visão holística. Ajuda o enfermeiro a organizar, tomar decisões, prever e avaliar. É a essência da prática de enfermagem, sendo uma atividade privativa do enfermeiro". (E12).

É guiar as ações de enfermagem, a fim de atender as necessidades individuais do paciente, família e comunidade. (TE03).

[...] um método de avaliação para se chegar à condição de saúde do paciente. (TE20).

Categoria 2 - Entendimento da equipe de enfermagem quanto aos benefícios da SAE para os pacientes

Sobre os benefícios, a equipe não demonstrou domínio da amplitude de benefícios que a SAE proporciona ao sistema de Saúde. O benefício mais citado foi a assistência individualizada pautada nas especificidades do paciente.

$\mathrm{Na}$ fala de uma das participantes, a aplicabilidade da SAE dependerá da patologia em questão, o que está em desacordo com a finalidade da profissão, pois segundo a Resolução 358/2009, o planejamento da assistência de enfermagem deve ser conforme as respostas biopsicossociais do paciente com a finalidade de otimizá-las e promover a resolutividade.
Seria o tratamento individualizado. (E5).

O paciente é melhor assistido e de acordo com suas prioridades. (E18).

São feitos os cuidados necessários para cada paciente dentro de sua patologia. (TE01).

Coletar dados e avaliar o estado de saúde do paciente. (TE02).

Entendimento dos benefícios da SAE como um método que organiza a assistência e dá qualidade nos cuidados de enfermagem

Para a equipe os benefícios da SAE para os cuidados de enfermagem, são a organização, qualificação, excelência na assistência.

Com a utilização da SAE, são assegurados não apenas os benefícios diretos ao cliente, como organização e qualidade no atendimento, mas também os benefícios voltados à instituição, como redução de gastos e diminuiç̧ão da internação hospitalar ${ }^{4}$.

Com a SAE podemos gerenciar o cuidado ao paciente, organizando o trabalho de assistência ao paciente. (E4).

Organização da assistência de enfermagem. (E5).

Devido agregar valor, padronização, unificação, quanto direção e qualidade na assistência prestada ao paciente. (TE03).

[...] possibilidade de organizar o trabalho e de elaboração de cuidados individualizados para atender as necessidades de cada cliente. (TE05).

Atendimento com mais qualidade. (TE19).

Os participantes sabem o conceito de $\mathrm{SAE}$, mas não sabem descrever os principais benefícios nas práticas assistenciais.

Além disso, há o descompasso entre a teoria versus a prática. Pois, apesar do conhecimento evidenciado pela equipe sobre SAE e a importância dela, muitos 
cuidados não são executados. De acordo com alguns participantes, isto ocorre, por conta que o enfermeiro não realiza a prescrição de enfermagem de forma adequada. Consequentemente, uma vez, que a normatização da assistência não ocorre, as práticas de cuidado tornam-se empíricas, o conhecimento científico é deposto e isso reduz as possibilidades da eficácia das intervenções planejadas.

Alguns técnicos relataram, ainda, que não há um instrumento adequado para preenchimento, não há apoio para a equipe, e o enfermeiro do plantão seguinte não dá continuidade no processo. Dessa forma, os cuidados de enfermagem tornam-se mecânicos e burocráticos, sem objetividade, despidos de criticidade e raciocínio clínico. E a consequência disso será a não compensação das respostas do paciente e o aumento da probabilidade de erros e eventos adversos na assistência.

\section{DISCUSSÃO}

Um dos passos fundamentais para a implementação da SAE e do Processo de Enfermagem (PE) nas Instituições é a credibilidade da equipe de enfermagem nesta ferramenta, anexa ao entendimento sobre a aplicabilidade e a viabilidade delas na assistência ${ }^{6}$.

A partir desse conhecimento e adesão é que os usufrutos da SAE serão colhidos, ou seja, o reconhecimento profissional só se dará a partir de conhecimento científico e domínio na utilização da SAE por parte do enfermeiro ${ }^{8}$.

E o que é identificado a partir desta pesquisa, bem como outras, é que há uma fragilidade no conhecimento da SAE por parte da equipe de enfermagem, ou mesmo, há um conhecimento, no entanto, ele é insípido e incapaz de modificar a realidade institucional e despido da capacidade de melhorar as respostas humanas do paciente, logo, as finalidades não são alcançadas ${ }^{5,6-8}$. E isso pode significar que o processo de trabalho neste ambiente é mecânico, burocrático, centralizado no cumprimento do preenchimento do impresso hospitalar do PE e ausente do processo crítico-reflexivo8. Tais fatores interferem negativamente na segurança do paciente e no perfil de morbimortalidade hospitalar ${ }^{9-11}$.

O conhecimento sobre a SAE e PE e seus benefícios é imprescindível para a melhor adesão da equipe de enfermagem ao instrumento e cria um ambiente favorável para sua implementação. Conhecer de fato todos os benefícios dela na assistência, para a visibilidade e autonomia profissional, como também para melhor dimensionamento dos recursos humanos e materiais, além de contribuição para otimização das respostas humanas do paciente, raciocínio clínico e tomada de decisões $^{5,12}$. Vale ressaltar que instaurar o PE de forma efetiva é cooperar junto ao programa nacional de segurança do paciente e a política nacional de humanização, já que esta ferramenta preza pela assistência individual e integral ao paciente através de um processo cíclico de coleta de dados, planejamento, implementação dos cuidados e avaliação ${ }^{10-11}$.

Na implementação da SAE todos são beneficiados: a instituição de saúde tem gastos menores com infecção relacionada a assistência a saúde, o índice de eventos adversos é menor, o tempo de internação é menor, o paciente tem suas respostas otimizadas, a equipe de enfermagem segue padronização e tem melhor usufruto do tempo e recur$\operatorname{sos}^{5,12-13}$. Mesmo com todos esses benefícios inúmeros estudos ressaltam as dificuldades na ratificação da $\mathrm{SAE}$, como falta de apoio do hospital, não conhecimento sobre o instrumento e seus benefícios ${ }^{5}$.

Esta cultura voltada a não valorização da SAE como instrumento de emancipação profissional começa por vezes na própria formação acadêmica, em que o estudante não é estimulado a pensar criticamente em questões específicas voltadas a essência da enfermagem, o que pode culminar em uma interpretação errônea sobre a SAE e o não entendimento das principais complexidades e desafios de sua implementação ${ }^{14}$. Quando esse preparo não é realizado e os desafios não são compreendidos, podem culminar na frustração pro- fissional, conformidade e incapacidade de tentar modificar a realidade ${ }^{12,14}$.

Um achado importante encontrado na pesquisa é que em nenhum momento foi citada as teorias de enfermagem na assistência, no entanto, de acordo com a resolução 358/ 2009 elas são importantes e indispensáveis como subsídio teórico para a $\mathrm{SAE}$, já que explicam os fenômenos relacionados a saúde e guiam o plano de cuidados ${ }^{1}$.

$\mathrm{Na}$ pesquisa foi evidenciado também que a prescrição de enfermagem não é clara e concisa para toda a equipe o que pode culminar em intervenções inadequadas com riscos de promover danos a saúde do paciente e eventos adversos, uma ocorrência constante em Instituições Hospitalares ${ }^{10}$. Tal fator ocasiona também um má registro da assistência de enfermagem, dificulta a avaliação e continuidade da assistência ${ }^{5}$.

$\mathrm{Na}$ equipe de enfermagem percebe-se que não há uma apropriação da SAE como instrumento profissional, pois a referência a ela no discurso dos participantes é revelada como longínqua e não há uma auto integração do participante neste processo. Especialmente, a fala dos técnicos de enfermagem expressam dúvidas acerca dessa ferramenta. Nesse contexto, é importante ressaltar que na resolução 358/2009 enuncia que o técnico de enfermagem participa da execução do PE, sob supervisão do enfermeiro, sendo necessário, portanto que o técnico conheça a importância da SAE e seja incluído neste processo ${ }^{2,6}$.

O enfermeiro é um líder de referência para a implementação da SAE, evidenciado na fala dos participantes percebe-se que além do apoio institucional é este profissional que impulsiona sua implementação, norteia a equipe quanto a instauração desse processo e proporciona a capacitação e solicitação de apoio institucional ${ }^{3,6-8,10}$.

É importante ressaltar que esse estudo possui limitações, foi realizado em apenas uma Instituição de Saúde, e o perfil caracterizado aqui pode ser atípico das demais instituições. Além disso, o foco da pesquisa foi o entendimento dos profissionais sobre $\mathrm{SAE}$, embora possam existir 
outros fatores que também podem contribuir para a não efetivação da SAE e para descrevê-los seria necessário uma análise funcional e multidimensional.

\section{CONCLUSÃO}

O resultado deste estudo demonstra que a equipe de enfermagem do presente local possui pouco entendimento sobre a SAE, sendo que houve maior apropriação do assunto pelos participantes, no que diz respeito ao conceito de SAE anexo a atribuição jurídica. No entanto, esses conceitos não foram vivenciados e alicerçados na assistência. Além de que, os benefícios supracitados pelos participantes são mínimos em contraposição ao legado de benefícios proporcionados pela SAE e PE em termos de sistema de saúde.

Portanto, não houve apropriação e incorporação deste processo pela equipe de enfermagem em termos de implemen- tação. Fator evidenciado pelas seguintes situações: comunicação inefetiva na passagem de plantão, não continuidade da assistência, falta de instrumento padronizado para os cuidados de enfermagem, falta de apoio dos gestores ao impulsionamento deste processo.

Logo, as consequências da não adesão a SAE e PE podem refletir em desfechos desfavoráveis a saúde do indivíduo: maiores chances de complicações, eventos adversos, maior tempo de internação hospitalar, maiores custos, deslocamento inadequado dos recursos materiais e humanos, procedimentos tecnológicos desnecessários, maiores transtornos na rotina do paciente e família e alterações psicoemocionais decorrentes do processo de hospitalização.

Especificamente, o não entendimento sobre SAE e a não aplicação no setor de maternidade implica em riscos para a segurança dos pacientes, por tratar-se de um local em que a díade mãe-bebê estão em processos de vulnerabilidade biológica e ambiental. Sendo, portanto, de responsabilidade da equipe de enfermagem prestar assistência livre de danos, respaldado cientificamente e sistematicamente em ferramentas profissionais imputadas pela Legislação, que nesse contexto encaixa-se a SAE e PE.

Dessa forma, este estudo corrobora com os demais ao trazer a essência de como a compreensão da SAE por parte da equipe de enfermagem pode favorecer ou desmerecer a implementação desta ferramenta. Como desafios principais evidenciados neste estudo frente as dificuldades de concretização da SAE estão burocratização dos serviços, mecanização, conformidade, não conhecimento sobre as etapas do PE pela equipe de enfermagem, falta de apropriação de conhecimento científico e planejamento. Podendo estes ocasionar inúmeras deficiências na assistência de enfermagem.

\section{Referências}

1. Conselho Federal de Enfermagem. Resolução COFEN no 358 de 15 de outubro de 2009. Dispõe sobre a Sistematização da Assistência de Enfermagem e a implementação do Processo de Enfermagem em ambientes, públicos ou privados, em que ocorre o cuidado profissional de Enfermagem, e dá outras providências. Rio de Janeiro: Conselho Federal de Enfermagem; 2009. Disponível em: <http://www.cofen.gov.br/resoluo-cofen-3582009_4384.html> 2. Tuani PCOS, Cristiane CFMR, Santos MB, Lima LF, Barreto FTC, Andrade LV, et al. Percepções de profissionais de enfemagem acerca da integração do técnico de enfermagem na sistematização da assistência. Esc Anna Nery. 2017 21 (2). Disponível em: <http://dx.doi.org/10.5935/1414-8145.20170035>.

3. Gouveia IMA, Sá VN, Sousa AA, Letícia SRL, Karine GMM, América MFS. Implementação e Implantação da Sistematização da Assistência de Enfermagem. Rev Enferm UFPE on line. 2018; 12 (4): 1174-8. Disponível em: <https:// doi.org/10.5205/1981-8963-v12i4a231030p1174-1178-2018>

4. Tâmara CG, Eli AKC, Felipe LP, Moreschi C. A equipe de enfermagem frente ao processo de implantação da Sistematização da Assistência de Enfermagem. Rev Enferm e Atenção à Saúde. 2016; 5 (2): 87-95. Disponível em: < http://seer.uftm.edu.br/revistaeletronica/index.php/enfer/article/view/1621>. 5. Souza RS, Raquel ALPA, Aparecida FO, Silva AO, Rozário MFBS, Patrícia GNP. Sistematização da Assistência de enfermagem na perspectiva da equipe. Enferm. Foco. 2016; 7 (2): 32-36. Disponível em: <http://revista.cofen.gov.br/ index.php/enfermagem/article/view/803>

6. Gaby MRG, Coelho SRVM. Systematization of nursing care and the formation of professional identity Sistematización de la Atención de Enfermería y formación de la identidad professional. Rev Bras Enferm. 2017; 70 (2): 455-60. Disponivel em: <http://dx.doi.org/10.1590/0034-7167-2016-0515>.

7. Farias TF, Matos E, Costa R, Chiodelli NS, Cristina IAM. Processo de enfermagem em centro obstétrico: Perspectiva dos enfermeiros. Texto Contexto Enferm . 2018; 27 (3): 2-10. Disponível em: < http://dx.doi.org/10.1590/0104$070720180004600016>$.

8. Ineu JD, Stein DB, Biazus CD, Danilo WLF, Crecencia HHS, Zamberlan C. Sis- tematização da Assistência de enfermagem: Ordem, desordem ou (re) organização?. Rev Enferm UFPE on line. 2017; 11 (10). Disponível em: < http:// pesquisa.bvsalud.org/enfermagem/resource/pt/bde-33055>.

9. Rosario GSM, Herdy VA, Pereira DR, Duarte AG, Vieira MS, Bertilla MLRB. Articulação entre Sistematização de Enfermagem e procedimentos operacionais: Perspectiva dos enfermeiros dos bancos de leite. Rev Enferm UFSM. 2017; 7 (2): 179-192. Disponível em: <http://pesquisa.bvsalud.org/enfermagem/resource/pt/bde-34367>

10. Nascimento GP, Neuma RDCA, Melo IB, Maria AUR, Bezerra LM, Maria JS. Relação entre Sistematização da Assistência de Enfermagem e segurança do paciente. Enferm. foco. 2017; 8 (2): 21-25. Disponível em: < http://revista. cofen.gov.br/index.php/enfermagem/article/view/985>.

11. Riegel F, José NOJ. Processo de enfermagem: implicações para a segurança do paciente em centro cirúrgico. Cogitare Enferm. 2017; 22 (4). Disponível em: $<$ https://revistas.ufpr.br/cogitare/article/view/45577>.

12. Inácio MS, Marilda ZRR, Souza FT, Helena SHC. Systematization of nursing care: challenges and features to nurses in the care management. Esc Anna Nery. 2015; 19 (1): 47-53. Disponível em: < http://www.scielo.br/pdf/ean/ v19n1/1414-8145-ean-19-01-0047.pdf>.

13. BRASIL. Lei $N^{\circ} 7.498$, de 25 de jun. de 1986 . Dispõe sobre a regulamentação do exercício da enfermagem, Brasília, DF, jun 1986. Disponível em <http:// www.planalto.gov.br/ccivil_03/leis/L7498.htm>.

14. Porfírio JS, Lúcia MG, Maris AP. Systematization of Nursing Care in undergraduate training: the perspective of Complex Thinking. Rev. Latino-Am. Enfermagem2015; 23 (1): 59-66. Disponivel em: <http://dx.doi.org/10.1590/0104$1169.0096 .2525>$.

15. Caroline AC, Vitor JS. Nurses' social representations of nursing care systematization Representaciones sociales de la sistematización de la asistencia de enfermería desde la perspectiva de los enfermeros. Referência. 2018; 4 (16) 139-146. Disponível em: <https://doi.org/10.12707/RIV17069>. 